\title{
Editorial: alternative energy systems: materials for nanofluids, electrochromics, photocatalysis, and thermoelectrics
}

\author{
Nitin Chopra \\ Editor-in-chief, Nanomaterials and Energy \\ Associate Professor, Department of Metallurgical and Materials \\ Engineering, University of Alabama, Tuscaloosa, AL, USA \\ Adjunct Professor, Department of Biological Sciences, University of \\ Alabama, Tuscaloosa, AL, USA
}

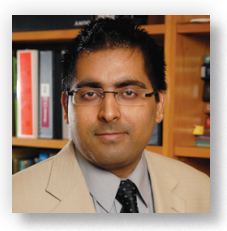

Innovation in nanofluids, electrochromics, photocatalysis and thermoelectrics is of immense interest. Although these four areas of alternative energy have been studied extensively, there remains a lack of robust material systems with suitable properties, energy structures and characteristics such as controlled thermal conductivity of nanofluids, efficient switching of an electrochromic material, modulated interfaces for photocatalysis and suitable figure of merit for thermoelectric systems. This necessitates understanding, development and modelling of materials and devices to obtain a synchronized set of data, which provides solid understanding of the relationship among structure, property, processing, and performance. This issue of Nanomaterials and Energy brings forward research articles in: (a) modeling of heat transport in a nanofluid system, $(b)$ growth mechanisms for thermoelectric materials, (c) electrochromism for solid-state devices, and $(d)$ composite photocatalytic architectures.

The first article, by Kosti, ${ }^{1}$ reports a heat transport and fundamental mathematical model explaining the effects of heat-flux boundary on a two-dimensional square enclosure using a copper-water nanofluid system. Governing equations have been modeled using the finite-difference technique. Important parameters of the study include Grashof numbers, nanoparticle concentration, and inclination angle. The authors also compare the results with previously published articles. Holder et al. ${ }^{2}$ report reaction mechanisms resulting in the growth of thermoelectric materials. The process described has potential for reducing manufacturing costs for such materials. The growth study demonstrates that, along with temperature and time of reaction, the reduction rate, conjugate anion of the starting reagent and the reaction atmosphere can influence the formation of thermoelectric materials. The article by Jain et $a l .{ }^{3}$ reports electrochromic behavior of a water-soluble polythiophene-based thin film device. The device demonstrates high stability and electrochromic switching from orange to green with high efficiency and fast switching times. The switching device could run for more than 50000 cycles. Finally, Tate and Johnston ${ }^{4}$ report a simple synthesis method to form novel silver/silver chloride (Ag/ $\mathrm{AgCl}$ ) nanocomposites using a porous support. The photocatalytic activity of silver-based polymer composites was evaluated by the photodegradation of methylene blue dye. The composite materials were shown to retain the enhanced photocatalytic activity displayed by $\mathrm{Ag} / \mathrm{AgCl}$ plasmonic photocatalyst nanoparticles.

With this issue, we welcome the audience of Nanomaterials and Energy to consider submitting their original research and enable cross-discipline sharing through this journal. This is also the last issue of 2014 and we wish our readers, authors, reviewers and editorial board members a very happy and prosperous 2015 . Nanomaterials and Energy is looking forward to publishing several themed issues and special feature articles in the coming year. In this regard, we encourage prospective authors and readers to consider suggesting ideas on the themed issues or about their interest to lead such a project. Nanomaterials and Energy also welcomes ideas for new opportunities from our authors and readers, provides a rigorous platform for sharing alternative energy research and strives to maintain high-quality publication standards.

\section{REFERENCES}

1. Kosti, S. Numerical study of heat-flux boundary in nanofluid-filled cavity. Nanomaterials and Energy 2014, 3 (6), 193-205.

2. Holder, C. F.; Rugen, E. E.; Anderson, M. E. Comparative growth mechanism study for two thermoelectric compounds. Nanomaterials and Energy 2014, 3 (6), 206-214.

3. Jain, V.; Yochum, H. M.; Montazami, R.; Heflin, J. R. Green colouring electrochromic devices of water-soluble polythiophene. Nanomaterials and Energy 2014, 3 (6), 215-221.

4. Tate, E. W.; Johnston, J. H. Photocatalytic silver/silver chloride polymer nanocomposites. Nanomaterials and Energy 2014, 3 (6), 222-228. 